\title{
Formação em Psicologia: Requisitos para Atuação na Atenção Primária e Psicossocial
}

\author{
Formation In Psychology: \\ Requirements For Acting In Primary \\ And Psychosocial Attention \\ Formación En Psicología: \\ Requisitos Para Actuación En La \\ Atención Primaria Y Psicosocial
}

Magda Dimenstein

Universidade Federal do Rio Grande do Norte

João Paulo Macedo

Universidade

Federal do Piauí
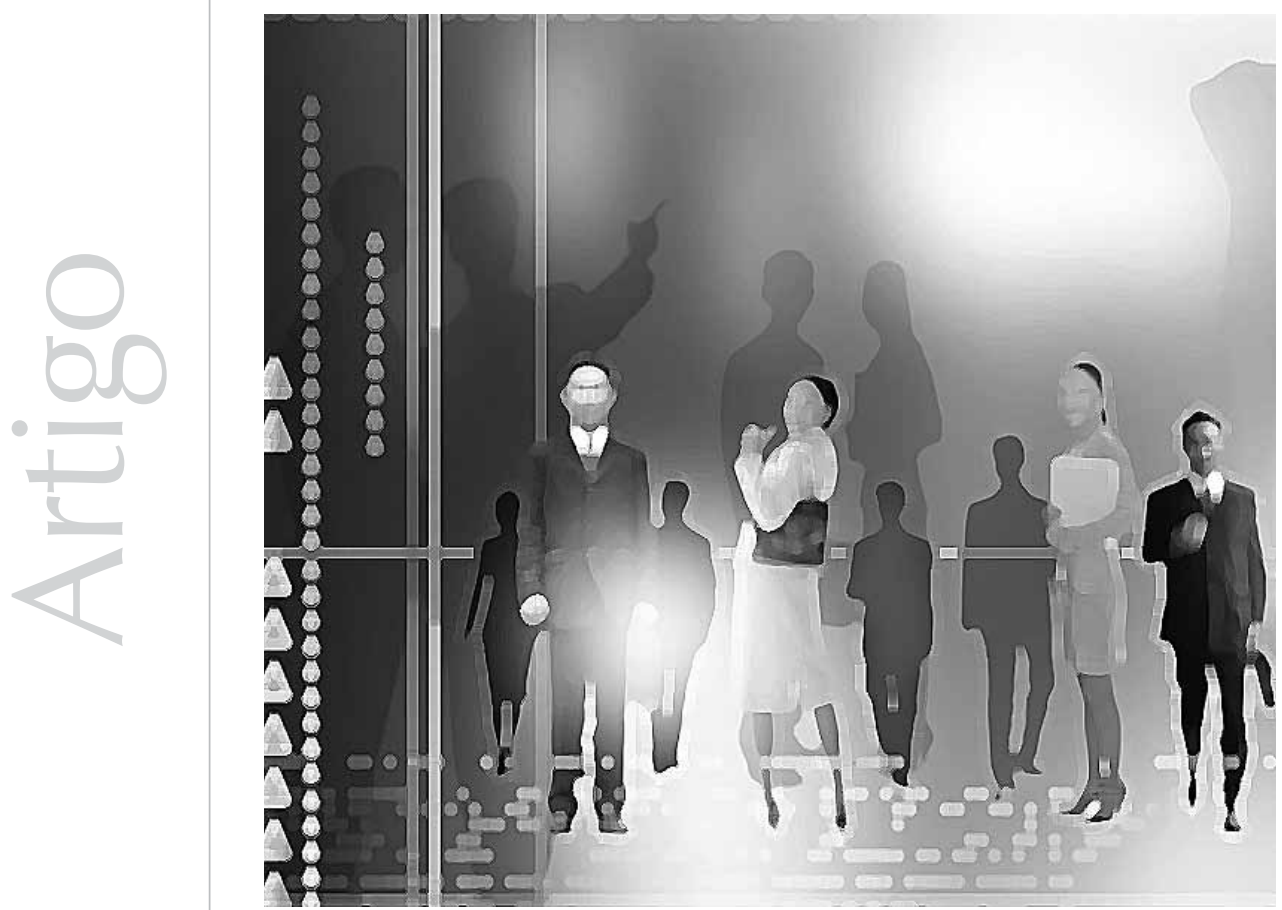
Resumo: Este artigo põe em análise o percurso profissional dos psicólogos na saúde pública nesses 50 anos de profissão no Brasil. Para tanto, destacam-se os estudos que versam sobre a formação, a inserção e a atuação dos psicólogos no SUS e os questionamentos surgidos em torno dos (des)encontros entre nossa ação profissional e as diretrizes/modos de trabalho preconizados na saúde coletiva. Entretanto, apresentamse algumas experiências em curso que têm conseguido provocar mudanças importantes na formação graduada e pós-graduada em saúde: residências multiprofissionais (RMS), Programas de Educação pelo Trabalho para a Saúde (PET-Saúde/MS-MEC) e programas de estágio profissionalizante em saúde coletiva em cursos de Psicologia. Conclui-se que a Atenção Primária e a atenção psicossocial que orientam o campo da saúde mental têm conformado uma conjuntura privilegiada para o desenvolvimento de experiências transformadoras, de novas competências e habilidades psicossociais, trazendo inovações para a formação e a requalificação dos modos de atuação do psicólogo no setor.

Palavras-chave: Formação do psicólogo. Sistema Único de Saúde. Atenção primária à saúde. Saúde mental.

Abstract: This article analyses the professional course of psychologists in public health in fifty years of the profession in Brazil. The studies that deal with formation, insertion and action of psychologists in SUS and the questioning that has arisen about the (dis)encounters between our professional action and the guidelines/methods of work preconized in collective health are highlighted. However, some experiences that have managed to cause important changes in undergraduation and graduation formation in health are presented: multidisciplinary residences, Programs of Education through Work for the Health and programs of professionalizing internship in collective health in psychology courses. The conclusion that the Primary Attention and the Psychosocial Attention that orientate the field of mental health have created a priviledged situation to the development of transformative experiences, bringing innovation to formation and requalification of the modes of the psychologists who work in the field.

Keywords: Psychologist education. Single Health System. Primary health care. Mental health.

Resumen: Este artículo pone en análisis el recorrido profesional de los psicólogos en la salud pública en esos 50 años de profesión en el Brasil. Para tanto, se destacan los estudios que versan sobre la formación, la inserción y la actuación de los psicólogos en el SUS y los cuestionamientos surgidos en torno de los (des) encuentros entre nuestra acción profesional y las directrices/modos de trabajo preconizados en la salud colectiva. No obstante, se presentan algunas experiencias en curso que han conseguido provocar mudanzas importantes en la formación graduada y posgraduada en salud: Residencias Multiprofesional (RMS), Programas de Educación por el Trabajo para la Salud (PET-Salud/MS-MEC) y programas de prácticas de formación profesional en salud colectiva en cursos de Psicología. Se concluye que la Atención Primaria y la atención psicosocial que orientan el campo de la salud mental han conformado una coyuntura privilegiada para el desarrollo de experiencias transformadoras, de nuevas competencias y habilidades psicosociales, trayendo innovaciones para la formación y la recalificación de los modos de actuación del psicólogo en el sector.

Palabras clave: Formación del psicologo. Sistema Unico de Salud. Atención primaria de salud. Salud mental.

Este artigo pretende percorrer as principais linhas problematizadoras sobre a presença dos psicólogos na saúde pública ao longo desses 50 anos de profissão no Brasil. Nossa intenção, nessa trajetória, é evidenciar alguns norteadores teórico-técnicos e políticos para a profissão, os quais são fundamentais para a garantia de uma formação mais condizente com os projetos da Reforma Sanitária e Psiquiátrica em curso no País.

De acordo com Fleury (2009), a reforma sanitária brasileira surge a partir de algumas crises vividas na década de 70, seja em relação ao conhecimento e à prática médica, à situação política e social expressa pelo autoritarismo e negação de direitos, às condições sanitárias em que vivia grande parte da população do nosso país, seja pela crise do sistema de prestação de serviços de saúde. Como projeto societário e movimento sociopolítico, a reforma sanitária foi "teorizada para alcançar a revolução do modo de vida" (Paim, 2008, p. 628) da sociedade brasileira e não apenas para realizar mudanças setoriais e institucionais, algumas vistas atualmente. Desse modo, compreendese a reforma sanitária como um projeto, um processo e um movimento de reforma social mais ampla que prevê: a) a garantia constitucional do direito universal à saúde, b) o reconhecimento dos determinantes 
sociais do processo saúde-doença, c) a luta pela constituição e reformulação de um campo de saber que respeita a diferença, pois pretende ser multiparadigmático, pluri e interdisciplinar e pluri e interprofissional, o qual marca uma nova ética profissional, d) a luta pela efetivação do Sistema Único de Saúde (SUS), que implica, além da ampliação e acesso universal e equânime dos usuários à rede de serviços, a reorientação das práticas e a criação de instrumentos de gestão democrática e de controle social em "direção à outra lógica que, por ter o usuário como central ao sistema de saúde, garante a exigibilidade de seus direitos, a humanização do acolhimento e a eficácia e a resolutibilidade do cuidado" (Fleury, 2009, p. 750).

O próprio processo de implantação do SUS tem sido marcado por inúmeros debates e correlações de forças para a sua consolidação como principal política social do País. Por estar inserido na seguridade social brasileira, ao lado da Assistência e Previdência Social, o SUS foi estruturado a partir de concepções e de princípios que tornam complexo o conceito de saúde, constituindo-o para além da ideia de ausência de doença, de conservação da vida ou de manutenção da sobrevivência. Saúde passa a ser entendida como um processo singular e subjetivo de negociação permanente de sentidos em um campo social, processo de construção e de desconstrução de normas para o enfrentamento da realidade e da (re)qualificação da vida.

Para dar conta da complexidade da vida e das suas necessidades, que envolvem tanto o bem-estar físico quanto os demais atravessamentos, sejam eles subjetivos, sociais, econômicos, culturais ou ambientais, que participam do processo saúde-doença, o SUS foi organizado em diferentes níveis de atenção, cujas estratégias de promoção, proteção, tratamento e reabilitação se ancoram na qualificação do processo de trabalho e no funcionamento dos serviços, bem como visam ao enfrentamento das desigualdades sociais e das necessidades específicas de saúde e à constituição de processos democráticos e de participação social (Feuerwerker, 2005).

Baseado nisso, temos assistido, nesses 20 anos de implantação do SUS, o movimento de expansão e interiorização, e em consequência, de "inversão do parque sanitário brasileiro" (Machado, 2006, p.12) quanto à oferta de serviços e às ações de cuidado, antes centrado na alta complexidade, agora na Atenção Primária em Saúde/APS, apesar das inúmeras carências ainda existentes no setor. Nos anos 80, contávamos com 18.489 estabelecimentos de saúde, com prioridade para os serviços hospitalares e ambulatoriais; já no final dos anos 2000, alcançamos o patamar de 96.450 estabelecimentos vinculados ao SUS (IBGE, 2009), com significativa quantidade de serviços e equipes de trabalho na Atenção Primária em Saúde.

A inversão na lógica sanitária no País é, sem dúvida, fruto do processo de municipalização do setor, pois, das 52 mil unidades assistenciais públicas em atividade, 95,6\% são municipais (IBGE, 2009); esse é, portanto, um cenário que demarca uma forte expansão das políticas setoriais, bem como de ações, serviços e programas vinculados ao SUS, por meio: a) da significativa ampliação e do fortalecimento da rede e das ações na atenção básica, através da Estratégia Saúde da Família (ESF), que articula equipes com o Núcleo de Apoio à Saúde da Família (NASF), além das equipes de Apoio Matricial e Unidades Básicas/ Centros de Saúde e Unidades Mistas, e b) do crescimento das equipes multiprofissionais nos serviços especializados, notadamente nos Centros de Atenção Psicossocial (CAPS), serviços residenciais terapêuticos, consultórios de rua, hospitais geral e especializado, serviços de referência em Medicina física e reabilitação e ambulatórios multidisciplinares 
especializados. A consolidação desses serviços por todo o País tem contribuído, sem sombra de dúvida, para o fortalecimento da presença do psicólogo no SUS (Böing, 2009).

Entretanto, o encontro da Psicologia com o SUS, especialmente com os serviços de atenção primária à saúde, bem como de saúde mental, tem aproximado nossa profissão de uma realidade ainda distante da que comumente conhecemos e discutimos em nossa formação e, em consequência, da que lidamos nos clássicos lugares de atuação. O encontro com comunidades, em geral de baixa renda e com problemas de infraestrutura, tem contribuído para o questionamento das nossas ferramentas de trabalho, do nosso aparato teóricotécnico e da efetividade de nossa atuação em um campo que demanda intervenções interdisciplinares por meio de equipes multiprofissionais.

Para compreendermos mais sobre os limites e as problematizações impostas pelo campo da saúde pública ao psicólogo brasileiro, discorreremos a seguir sobre o movimento de inserção e de atuação da nossa categoria profissional no SUS e os questionamentos surgidos em torno dos (des)encontros entre a ação profissional dos psicólogos e as diretrizes/modos de trabalho no setor.

\section{Inserção e atuação do psicólogo no SUS}

A entrada dos psicólogos no campo da saúde pública guarda uma relação estreita com os rumos tomados pelo movimento de Reforma Sanitária e Psiquiátrica ocorrido no Brasil a partir de meados dos anos 70, momento no qual se instaurou uma crítica ao projeto privatista de cuidados em saúde, cuja ênfase recaía no aspecto curativo, individual, orientado para o lucro e para a privilegiação do produtor privado desses serviços (Mendes, 1994, p. 22). Antes disso, algumas poucas experiências foram desenvolvidas no campo da saúde, quando os psicólogos ingressaram em equipes multiprofissionais em hospitais privados e públicos ligados às escolas de saúde de São Paulo e Rio de Janeiro. O objetivo desses profissionais nessas equipes era o apoio às internações clínicas e/ou cirúrgicas em pediatria e cardiologia, dentre outros. No entanto, foi na saúde mental que a entrada dos psicólogos no setor público se fez mais vigorosamente. Com o surgimento dos primeiros movimentos de mudança do quadro precário de assistência psiquiátrica no País, a partir de investimentos de serviços substitutivos ao manicômio, como também com a formação e a contratação de pessoal capacitado para operar o novo modelo de assistência em saúde mental, abriu-se um vasto campo de trabalho para o psicólogo nesse contexto, que atribuía às equipes multiprofissionais um papel fundamental de transformação das velhas estruturas e como condição sine qua non para a concretização dos serviços extra-hospitalares tais como os centros e os ambulatórios de saúde. O psicólogo passou a ser considerado um profissional capaz de contribuir para a promoção da saúde mental, na medida em que tinha acesso a um instrumental teóricoprático que poderia ser de grande utilidade na identificação e na abordagem de situações consideradas de risco, ou seja, propiciadoras de transtornos mentais. Dessa forma, o psicólogo poderia intervir precocemente junto àqueles fatores considerados insalubres para a saúde mental - nos moldes da psiquiatria preventiva norte-americana dominante na época - especialmente nos momentos críticos da vida das pessoas, e assim desenvolver uma prática psicoterápica dirigida para a prevenção e em benefício da comunidade.

Nota-se, então, a manifestação de um interesse pelo psicólogo por parte dos órgãos públicos, o que implicou um movimento de contratação desse profissional, especialmente 
nos níveis estadual e municipal. Esse movimento de absorção de psicólogos no campo da saúde pública observado no final dos anos 70 intensificou-se ainda mais na década seguinte, marcada por políticas públicas de saúde que priorizavam a criação de equipes multiprofissionais como forma de melhorar a qualidade dos atendimentos e de valorizar o profissional de saúde. Essa tendência a atribuir às equipes um papel decisivo no processo de reforma do sistema de saúde foi o que fez com que muitos profissionais, antes alheios ao campo da saúde pública, passassem a fazer parte dos quadros funcionais. Os psicólogos, como uma das categorias que mais se beneficiou com esse movimento, foi a categoria que teve o maior número de profissionais contratados ao longo das últimas décadas para trabalhar nas instituições públicas. Em 1976, contávamos com 723 psicólogos nas equipes de saúde em todo o Brasil; em 1984, esse número chegou a 3.671 profissionais (Machado, Médici, Nogueira, \& Girardi, 1992), e, atualmente, existem mais de 40 mil profissionais vinculados ao SUS ${ }^{1}$.

No final da década de 80, com os avanços no processo de desinstitucionalização psiquiátrica, observa-se a ênfase no desenvolvimento de práticas inovadoras de cuidado e de assistência em função de um conceito mais ampliado e complexo de saúde mental, o que exigiu modelos efetivamente transdisciplinares de integração de saberes. Houve a extensão da rede ambulatorial e a formação das equipes multiprofissionais, passando a Psicologia a fazer parte de um conjunto de saberes que buscava romper os limites da concepção tradicional de saúde mental e das práticas assistenciais asilares não só no setor secundário, mas também no setor primário em saúde.

$1 \mathrm{http}: / /$ cnes.datasus. gov.br/Mod_Ind Profissional com CBO. asp?VEstado $=\overline{0} 0 \&$ VMun $=$
Paralelamente a esse momento político de instauração de programas com ênfase na questão ambulatorial e na montagem multiprofissional das equipes de saúde, a Psicologia vinha sendo alvo de inúmeras críticas no sentido de que o trabalho clínico tradicional do psicólogo não apresentava grande significado social, pois, destinado a uma pequena minoria da população, despreocupava-se com os problemas sociais, além de estar impregnado de forte conteúdo ideológico individualista. Para Gil (1985), a inserção na saúde pública foi, para o psicólogo, a maneira de ter uma função socialmente reconhecida e a estratégia para escapar ao declínio social que a categoria vinha experimentando. Esse declínio foi intensificado pela forte recessão da classe média nos anos 80, grande consumidora das práticas psicológicas, cujas famílias vivenciavam a falência dos seus projetos de ascensão social, típicos dos modelos familiares nucleares e expressivos da modernidade individualizante. A Psicologia, na medida em que não dava respostas satisfatórias às questões sociais cotidianas, foi perdendo sua legitimidade social, o que impulsionou os profissionais a buscar novas formas de inserção no mercado de trabalho.

Não foi por acaso que fortes investimentos no campo da saúde pública foram operados pelo Conselho Federal de Psicologia nas últimas décadas, forçando a construção de uma identidade profissional atrelada à saúde, considerada não mais como área específica de interesse do psicólogo, mas "como um dos importantes espaços de referenciação do diálogo da Psicologia com a sociedade" (Conselho Federal de Psicologia - CFP, 2006, p. 6). Isso significou não só a disputa por espaço no concorrido mercado de trabalho para os profissionais de saúde que integram o SUS mas também investimentos estratégicos tanto em termos da abertura de novos cursos e da sua redefinição política e pedagógica, tentando dar respostas aos projetos, programas e serviços ligados às políticas públicas (saúde, saúde mental, assistência social, luta pela terra, instalação 
da rede de proteção de direitos, etc.), quanto de capilarização e interiorização da Psicologia para as cidades de pequeno e médio portes, invertendo a tendência eminentemente urbana da profissão (Macedo \& Dimenstein, 2011).

Assim, há pelo menos 20 anos, observamos movimentos na categoria em torno da construção desse novo lugar social para a Psicologia e da ampliação da sua presença no campo das políticas públicas e práticas sociais, sob a forte argumentação da nossa inerente "vocação para a promoção do bem-estar e ampliação da qualidade de vida dos indivíduos, dos coletivos e das instituições" (CFP, 2006, p.4). Deu-se, portanto, a invenção de um novo operador no campo da saúde pública, em especial no da saúde mental, bem como a produção da legitimidade da categoria por meio de uma intensa negociação com o Estado brasileiro pela ampliação da inserção da Psicologia no SUS, que passou a ser um mercado estável para a área. Essa nova perspectiva tornou-se não só um dos elementos mais significativos do processo de remodelamento posto em marcha pela categoria como também um dos mais eficazes em qualificar formas de vida, em definir tecnologias para atuar sobre ela e em intervir na resolução de problemas e na correção dos desajustes e desvios identificados nas engrenagens sociais em nome da promoção da saúde. Como aponta Bernardes, "a Psicologia oferece para a saúde pública a interioridade do sujeito público e a racionalidade técnica para seu governo" (2010, p. 214).

Não é desconhecido que essa dimensão de controle e governo dos corpos e da vida é constitutiva da história da Psicologia, que nasceu e se perpetuou, em nome da razão, comprometida com a produção de saberes e técnicas objetivas e neutras voltadas para processos de adaptação dos indivíduos às normas sociais, tomadas como naturais e a-históricas, servindo, dessa maneira, de suporte científico das ideologias dominantes, das desigualdades e da exclusão social.

Nesse caso, é preciso reconhecer que a participação dos psicólogos no SUS tem pelo menos dois aspectos que consideramos fundamentais. O primeiro deles é que a presença desse profissional nos serviços e nas equipes de saúde em todo o País não implicou necessariamente mudança radical na lógica de produção de saberes e de atos de saúde pela própria categoria, bem como no agir diário dos outros profissionais. Nesse aspecto, chamamos a atenção para o fato de o psicólogo ser mais um dos atores partícipe no variado e contraditório bloco de forças que compõe o território da saúde pública, que atua orientado por concepções biologizantes e mecanizadas da vida, por um caráter prescritivo em termos dos modos de existência, por uma tendência ao trabalho individualizado, ancorado em relações verticalizadas e não focadas no usuário, por intervenções pouco variadas, circunscritas a queixas e voltadas para a remissão de sintomas, bem como por estratégias de subjetivação norteadas pelos princípios da disciplina, da normatização e da cristalização das referências identitárias.

Essa lógica orienta as ações, define técnicas e procedimentos, organiza o cotidiano do trabalho e suas rotinas, seleciona a clientela, faz declinar a participação e o envolvimento nos assuntos coletivos, enfim, é o que tem conformado o modelo hegemônico de atuação do psicólogo no SUS do ponto de vista tecnoassistencial e ético-político. Não por menos, o próprio padrão de funcionamento dos psicólogos no SUS tem sido alvo de duras críticas. A vasta literatura nacional vem caracterizando as práticas dos psicólogos nas instituições públicas de saúde, em particular, na rede de atenção primária e de saúde mental como permeada de dificuldades, limites e desafios 
no sentido de produzir novos modos de atuação condizentes com os princípios e as diretrizes do SUS e da Estratégia de Atenção Psicossocial, apesar de algumas tentativas de mudanças na formação acadêmica e profissional (Benevides, 2005; Yamamoto, 2007; Guareschi, Scislesk, Reis, Dhein, \& Azambuja, 2010; Scarcelli \& Junqueira, 2011, dentre outros).

Por outro lado, existe atualmente um campo de tensionamento importante que extrapola o âmbito da Psicologia e que é composto pelos diversos saberes que constituem a saúde coletiva ${ }^{2}$, e que tem questionado, entre outros, o valor da contribuição da própria Psicologia para o projeto político do SUS. Tais tensionamentos têm ajudado na formulação de novos problemas e constituído o segundo aspecto, que é a emergência de um ator político que intervém em um plano ético, ou seja, que provoca análises do que está posto em funcionamento, inclusive das próprias políticas públicas e os modelos tecnoassistenciais em curso, bem como das relações interprofissionais que reconhece que o cuidar em saúde não se restringe a competências e tarefas técnicas, mas prolonga-se para um nítido deslocamento de horizontes normativos, a partir dos projetos de felicidade de cada pessoa, que é aquilo que as move e que as identifica em seu existir concreto (Ayres, 2001).

Reinventar práticas e produzir-se profissional como um ator que põe em análise o que realiza supõe, operacionalmente, produzir rupturas na cultura de atenção aos usuários, nas modalidades de intervenção hegemônicas, bem como alterar os modos de organização, gestão e subjetivação nos serviços. Dessa maneira, os problemas, no âmbito do SUS, dizem respeito a uma complexa teia de determinação que envolve elementos macro e micropolíticos, indo além da prestação de práticas biomédicas e da oferta de serviços diversificados, dentre eles, o atendimento psicológico. Assim, é preciso voltar-se para uma dimensão ampliada que incorpora a complexidade subjetiva dos atores envolvidos, pensar na saúde como produção de subjetividade.

No campo da saúde mental, a contribuição dessa perspectiva tem sido no sentido de dar visibilidade a um conjunto de forças que operam visando a reduzir a experiência da loucura a uma patologia ou doença mental. Por meio da análise de discursos, práticas e instituições, podemos identificar mecanismos que buscam controlar, tutelar, normatizar e medicalizar a loucura, mas também inventar novos saberes e dispositivos em direção à desinstitucionalização da cultura manicomial, que vai muito além do âmbito restrito de serviços e práticas profissionais e diz respeito aos modos de vida tecidos no cotidiano, às formas hegemônicas de sociabilidade e de convívio com a diferença na contemporaneidade. Portanto, as lutas que travamos como coletivo de trabalho operam em diferentes planos, na concretude do presente, na materialidade dos discursos e práticas que forjam verdades amplamente divulgadas e consumidas e que, atualmente, estão cada vez mais interessados na chamada normalidade.

Contudo, o mais desafiante não é só problematizar noções e práticas e propor novos enfoques para a atuação do psicólogo no campo da saúde coletiva, mas dispornos à experimentação, ativar a ousadia de diferir e fazer com que esses conceitos ganhem um sentido prático, afastando-nos da inclinação ao congelamento, desinvestindo práticas idealizadas e o engessamento por referenciais transcendentes e universalizantes, tornando-nos "superfície tensionante e tensionada, dimensionada para hospedar novas plataformas de lançamento da vida" (Fuganti, 2008, p.17). 


\section{Construindo alguns norteadores para a formação profissional dos psicólogos}

Estudiosos de todo o País são unânimes em afirmar que a Psicologia é hoje uma das profissões que têm, ao mesmo tempo, forte inserção e potencialidade de contribuição para o projeto político do SUS, assim como sérios problemas no que diz respeito às práticas, seja em serviços técnicoespecializados, em estabelecimentos ou programas de cuidado à saúde e proteção de direitos, seja nas demais atividades gerenciais e de regulação dessas políticas. Entre as principais críticas, cita-se: manutenção do clássico modus operandi de atuação clínica liberal-privatista, de cunho individual e curativista, busca de nexos causais para a compreensão do quadro clínico e psicossocial do paciente, baseado unicamente nas definições de normal e patológico com foco na remissão dos sintomas, no fortalecimento da adesão ao tratamento prescrito e na normalização do paciente, na pouca atenção às necessidades sociais e de saúde que circunscrevem as queixas da população que procura os serviços, realização de ações profissionais isoladas, justificadas pelo respeito ao sigilo profissional, por isso a esquiva para a atuação em equipe, trabalho técnico dissociado das ações de gestão, seja ele realizado no próprio serviço, por meio de ações de gestão da clínica e do cuidado, seja ele voltado para a estrutura e a organização de sistemas de serviços e de redes integradas de saúde.

Nesse caso, percebe-se uma tendência quanto ao fato de que as atividades e os modos de intervenção dos psicólogos nos serviços de saúde são basicamente os mesmos, independentemente do local de atuação, da população atendida e das necessidades e demandas requeridas. Esse modelo tem forte relação com o "desinteresse, a alienação, o agir mecânico e burocratizado" (Campos, 1994, p.43) que tanto caracteriza a presença do psicólogo no SUS como um profissional que não sabe diferenciar e qualificar suas práticas de acordo com o nível de atenção (primário, secundário ou terciário) em que está inserido.

Por outro lado, sabemos que a participação dos psicólogos no campo da atenção primária e no da saúde mental tem conformado uma conjuntura privilegiada que tem permitido o desenvolvimento de experiências transformadoras, de novas competências e habilidades psicossociais, trazendo inovações para a formação e a requalificação dos modos de atuação no setor, incrementando o protagonismo dos profissionais no campo da reforma sanitária e psiquiátrica. Diariamente, diversas demandas em saúde mental são identificadas por profissionais das equipes ESF e NASF. São situações que requerem intervenções imediatas, na medida em que podem evitar a utilização desnecessária de recursos assistenciais mais complexos. Tratase de problemas associados ao uso prejudicial de álcool e de outras drogas, aos egressos de hospitais psiquiátricos, ao uso inadequado de benzodiazepínicos, aos transtornos mentais graves e a situações decorrentes de violência, desfiliação e exclusão social. A identificação e o acompanhamento dessas situações, incorporados às atividades que as equipes de atenção primária desenvolvem, podem subsidiar ações de intervenção precoce, uso racional de medicamentos, continuidade dos cuidados, prevenção das admissões impróprias em hospitais psiquiátricos e estabelecimento e manutenção de sistemas de apoio comunitário.

Apesar dos inúmeros problemas observados que envolvem a implantação, a capacidade resolutiva, a política de pessoal e a forma de vinculação da clientela, os quais levam Campos, Gutiérrez, Guerrero e Cunha a reconhecer que "estamos longe de dispor 
de uma rede de atenção básica com ampla cobertura e com eficácia adequada" (2010, p. 144), não restam dúvidas de que os princípios e as diretrizes que organizam tanto a atenção primária, via ESF e NASF, quanto a Estratégia da Atenção Psicossocial/EAP, que orienta a Política Nacional de Saúde Mental, são capazes de disparar mudanças efetivas no modo de produzir ações em saúde, de fazer gestão, de reflexão e de proposição de soluções criativas para os problemas que permeiam o cotidiano do trabalho nesses campos.

Nesse aspecto, a formação acadêmica e profissional dos psicólogos precisa nutrir-se desse debate até mesmo para contribuir para o desenvolvimento de um estatuto diferenciado da nossa profissão no campo da saúde. Tanto a ESF quanto a EAP abrem uma nova agenda em termos do trabalho em saúde, dos modos de ser trabalhador no SUS, das competências requeridas, do compromisso ético-político com a produção de um sistema universal e resolutivo, associado à ideia de cidadania, que exige compromisso, sensibilidade, capacidade de negociar e de lidar com incertezas, bem como com problemas complexos e com a alta vulnerabilidade social que marca a realidade de grande parte dos usuários desses serviços. Porém, tudo isso precisa ganhar força nas formações dos psicólogos.

A multiprofissionalidade, o trabalho no território e em rede, articulado por equipes de referência que se responsabilizam pela condução e pelo acompanhamento dos casos, o trabalho a partir de uma perspectiva ampliada da clínica, não restrita a aspectos biomédicos, voltada para o segmento longitudinal com responsabilidade e vínculo, além da busca de obtenção de outros gradientes de autonomia na atividade profissional com a proposta da gestão compartilhada e participativa visando à não padronização das atividades, a aposta na articulação de diferentes saberes e tecnologias na construção de diagnósticos e projetos terapêuticos singulares, bem como o desenvolvimento de ações de aumento do coeficiente de autonomia dos usuários, ou seja, sua capacidade de "compreender e agir sobre si mesmo e sobre o contexto, lidando com sua rede de dependências" (Figueiredo \& Furlan, 2010, p.165), todos esses aspectos são norteadores importantes para qualificar a presença dos psicólogos no SUS e para superar sua história de desconhecimento dos aspectos organizacionais e institucionais, bem como a pouca bagagem para encarar os enormes desafios clínico-institucionais colocados à sua frente.

Nesse caso, a formação e o exercício profissional dos psicólogos não podem ficar alheios ao debate de estruturação do cuidado e dos novos modos de gestão dos processos de trabalho no SUS (Ferreira \& Olschowsky, 2010), primeiro, porque é insustentável a ideia de que os processos de formação estão deslocados ou ausentes dos acontecimentos do mundo e do cotidiano do serviço, que é, ao mesmo tempo, plano de reprodução, de experimentação e de invenção de modos de ser trabalhador no SUS (Neves \& Rollo, 2006); segundo, porque é preciso aprofundar a relação ensino-serviço-comunidade de modo que a formação se dê no e para o serviço, além de criar espaços coletivos em que os trabalhadores, os alunos-estagiários e os professores-orientadores possam, em conjunto, interrogar e analisar o seu cotidiano de trabalho na medida em que exercitam ações em equipe e potencializam a multiprofissionalidade, bem como a interdisciplinaridade norteada pelos cuidados integrais (Silva \& Caballero, 2010); por fim, que a base metodológica da proposta visa a articular "os componentes de gestão, assistência e participação popular, mobilizando a incorporação dos trabalhadores como atores identificados com as necessidades de criação e modificação no cenário da saúde" (Oliveira \& Gaureschi, 2010, p. 95). 
Dentre as principais experiências que têm conseguido provocar tais mudanças relativas à formação em saúde, destacam-se as residências multiprofissionais e os Programas de Educação pelo Trabalho para a Saúde ou Saúde Mental (PET-Saúde/MS e MEC). Parte do processo de Educação Permanente em Saúde/EPS, as residências multiprofissionais, que têm o caráter de formação pós-graduada, emergem como cenário de inspiração para formar profissionais com capacidade de desenvolver práticas convergentes com as diretrizes do SUS. As residências tomam os serviços e as equipes como espaços privilegiados de formação, de campos de atuação vivencial e de atualização contínua para seus profissionais, possibilitando o aprendizado coletivo no trabalho, mais próximo da realidade a ser considerada no planejamento e na atenção em saúde, e superam a ideia de desenvolvimento da tecnologia e do trabalhador como recurso, para o investimento em habilidades no trabalho em equipe, para construir novos pactos de convivência nos quais o envolvimento ético-político do trabalhador é o centro do processo pedagógico.

Essa perspectiva de articular ações intersetoriais na triangulação serviço-comunidadeAcademia, de abrir a universidade às demandas sociais para interesses e objetivos concretos das comunidades e serviços, redefinindo seu papel e sua responsabilidade social a partir de uma nova concepção do processo ensino-aprendizagem, que valoriza o saber prévio dos alunos e dos técnicos, e de uma postura problematizadora da realidade é a mesma que orienta as propostas PET e as de estágios em atenção primária e saúde mental que envolvem alunos de Psicologia. Apesar de ser um dos cursos integrantes e com maior número de profissionais nas RMS e PET, não há ainda uma vasta discussão, nem estudos avaliativos acerca da participação da Psicologia e de seus efeitos em termos das práticas e da cultura profissional da categoria.
Compartilhamos aqui duas experiências de ensino que reúnem alguns requisitos que consideramos imprescindíveis à formação de recursos humanos para a saúde coletiva, de modo a superar o tradicional papel das instituições formadoras de produzir e formular "conhecimentos que sustentam paradigmas tecnicistas e cartesianos, produtores de procedimentos e não de processos de cuidado" (Ceccim, 2010, p. 20). A primeira delas denomina-se $\mathrm{SACl}$ (saúde e cidadania), é realizada na UFRN e estende-se a todos os estudantes da área de saúde, incluindo a Psicologia. A segunda é relativa ao Programa de Estágio em Saúde Coletiva do Curso de Psicologia da Universidade Federal do Piauí, localizado no campus de Parnaíba. Ambas estão construídas na interface ensinoserviço-comunidade e pretendem conhecer e trabalhar com alguns problemas concretos e complexos da realidade local, assim como em espaços multiprofissionais. Para tanto, baseiam-se em novos modelos acadêmicos (novas metodologias de ensino, organização de conteúdos e cenários de aprendizagem) que, por sua vez, estão orientados por uma concepção crítica, reflexiva, sustentada na construção do conhecimento a partir da problematização da realidade, na articulação teoria-prática e na participação ativa do aluno no processo de aprendizagem.

Apesar de operarem em realidades distintas, essas experiências formativas compartilham alguns princípios norteadores da atuação do psicólogo na saúde pública. Nesse sentido, consideramos que estão de acordo com as recomendações da Organização Mundial da Saúde (OMS) para a saúde mental no mundo quando apontam a relevância da Atenção Primária à Saúde (APS) na promoção de saúde mental nas comunidades. No Brasil, nota-se um esforço nessa direção a partir da proposição da Política Nacional de Saúde Mental, em vigor há 10 anos. Em se tratando de uma política pública de base comunitária e territorial, 
a PNSM visa, a partir da coordenação, colaboração e complementariedade dos dispositivos e recursos sanitários e sociais, ao desenvolvimento de serviços que atendam as amplas necessidades e problemáticas sociais da população portadora de transtornos mentais, especialmente no que diz respeito à reabilitação psicossocial, ao apoio à integração laboral, à atenção residencial e ao suporte às famílias. Busca-se, por meio de tais estratégias, evitar processos de deterioração das capacidades e do funcionamento social das pessoas com transtornos mentais que acirram as situações de abandono e exclusão, bem como fomentar possibilidades de autonomia e de participação na vida comunitária.

No intuito de produzir interferências na lógica assistencial que nos impede de dispor de uma rede de atenção primária com ampla cobertura e com eficácia adequada em termos de saúde mental, o Ministério da Saúde vem desenvolvendo um conjunto de ações e de atividades no qual se insere o Programa Nacional de Melhoria do Acesso e da Qualidade da Atenção Básica - PMAQ (Brasil, 2012), como uma das principais estratégias indutoras de qualidade, que vem a ser o principal desafio do SUS. Para tanto, desenvolveu uma ferramenta que aborda diferentes áreas, dentre as quais a de saúde mental. Neste trabalho, ressaltamos os aspectos dessa ferramenta que têm o potencial de facilitar a mobilização de iniciativas para a mudança e o aprimoramento dos serviços, tarefas que tocam diretamente ao psicólogo nesse campo. Nessa perspectiva, interessa-nos ressaltar que, para uma atuação apropriada à $\mathrm{AP}$,

1) o psicólogo deve ser capaz de observar o contexto e de conhecer o território da área de abrangência da unidade de saúde considerando: a) a história do lugar, b) os aspectos geofísicos, situando os fatores ambientais de risco ou vulnerabilidade, c) os aspectos estruturais em termos da rede de esgoto, energia, água e esgoto, coleta de lixo, tipo de moradia, transporte, população, escolas, creches, demais equipamentos de saúde e de assistência social, equipamentos de lazer e igrejas;

2) o psicólogo deve conhecer os aspectos sociopolíticos em termos de grupos organizados, de lideranças (reconhecidas pela comunidade), de principal fonte de renda das famílias, de eventos significativos para a comunidade, e deve participar de ações de identificação e de enfrentamento dos problemas sociais de maior expressão local, com ênfase no desenvolvimento comunitário e na atuação intersetorial;

3) o psicólogo deve conhecer os principais problemas e necessidades de saúde da comunidade que vive na área de abrangência com base no seu perfil epidemiológico bem como se dá o acesso aos serviços e programas de saúde e/ou benefícios sociais, aos medicamentos, às práticas de cuidado desenvolvidas pelos profissionais de saúde (ESF) e às práticas integrativas (uso de fitoterápicos, rezadeiras, prática de atividades físicas); o profissional deve realizar atendimentos programáticos ou de livre demanda, dentro e fora do espaço físico da Unidade Básica de Saúde e nas visitas domiciliares, bem como outras estratégias de cuidado baseado na compreensão aprofundada dos fenômenos psicológicos e psicossociais que envolvem processos de saúde e de produção da vida em contextos comunitários;

4) esse diagnóstico local e situacional deve ser realizado com um processo de discussão e de negociação com os profissionais do serviço de saúde e com a comunidade, no sentido de estabelecer um consenso sobre os problemas prioritários para, em seguida, proceder à escolha das estratégias de intervenção participativas; para tanto, reúnese com a comunidade para desenvolver ações 
conhecer mais detalhadamente essas experiências, ver Medeiros Junior, A., Liberalino, F. N., \& Costa, N. D. L. (Orgs.) (2011).

4Instrumento de avaliação e de intervenção terapêutica, realizadas por meio de representação gráfica, que integra a história do processo saúde-doença e os processos psicossociais e comunicacionais que envolvem a dinâmica familiar e intergeracional (Brasil, 2011).

5Instrumento que complementa o genograma na avaliação das relações familiares com o meio social (sistema ecológico), permitindo que os padrões organizacionais e suas relações com o território sejam avaliados em termos de recursos e necessidades (Brasil, 2011). conjuntas e debater os problemas locais de saúde, o planejamento da assistência prestada e os resultados alcançados, disponibilizando informações sobre o funcionamento da unidade de saúde de maneira clara e acessível aos usuários;

5) a principal estratégia de cuidado é o estabelecimento de confiança e vínculo com as famílias e comunidades, por isso, conhecer de perto os problemas, as dificuldades, as histórias e projetos de vida, as necessidades sociais e de saúde, bem como as potencialidades das famílias, é imprescindível para o planejamento das ações e de um plano de cuidados continuados;

6) é necessário utilizar diversas ferramentas para o desenvolvimento do trabalho, dentre as quais se destacam a entrevista diagnóstica, de modo a subsidiar os estudos de casos e a elaboração de projeto terapêutico singular com base nas necessidades sociais e em saúde, as ações de acolhimento individual e coletivo, o acompanhamento de casos na perspectiva da gestão do cuidado, a orientação psicossocial, o acompanhamento terapêutico e a busca ativa em saúde mental, a visita domiciliar e o uso de ferramentas como o genograma ${ }^{4}$ e o ecomapa ${ }^{5}$;

7) é prioritário o desenvolvimento de atividades de apoio matricial e de articulação com a rede, fortalecendo ações de saúde mental na atenção primária. Além das tecnologias relacionais de vínculo e escuta, o uso do método da roda nas práticas grupais como intensificador de espaços coletivos para a produção de sujeitos corresponsáveis no processo saúde-doença, a identificação de temas e prioridades para o desenvolvimento de ações para a melhoria das condições de vida e sanitária do território, bem como demais estratégias de intervenção individual e coletiva são as principais ferramentas utilizadas na gestão da clínica pelo apoio matricial;
Por outro lado, para uma atenção nos casos de saúde mental, o psicólogo deve investir em ações de cuidado e reabilitação psicossocial, assumir a coordenação do cuidado e desenvolver ações que incluam acolhimento, tratamento e acompanhamento, prevenção primária ao uso prejudicial do álcool e outras drogas, diagnóstico precoce, redução de danos, reinserção social e referenciamento para a rede de atenção psicossocial, quando necessário. As ações nos dispositivos de saúde mental se concentram no desenvolvimento de atividades de acolhimento/triagem, no estudo de caso em equipe para a elaboração do projeto terapêutico singular, na realização de visita domiciliar, na construção de ações de retaguarda com atividades de interconsulta para os casos de internação, bem como de suporte à família, na realização de trabalho com grupos e participação das oficinas terapêuticas, com foco na autonomia e no desenvolvimento de ações de suporte e ajuda mútua, na participação nas ações de matriciamento na atenção básica e também na realização de grupos de promoção de saúde mental na atenção primária à saúde e pactuação da continuidade do cuidado no território de forma corresponsabilizada.

\section{Conclusão}

Apesar dos avanços quanto à ampla abertura de serviços que contam com a presença do psicólogo no SUS, não são poucas as dificuldades para organizarmos nossas ações profissionais com base na proposta da Reforma Sanitária e Psiquiátrica. Entretanto, já não é suficiente reconhecer que o psicólogo trabalha isoladamente, que opera predominantemente com psicoterapias, que tem pouco conhecimento do território onde atua, que acredita na neutralidade de seus saberes e práticas.

O desafio que se coloca não está simplesmente limitado a operar certos ajustes na formação acadêmica e profissional, ampliando o leque 
de disciplinas e conteúdos relacionados ao SUS e à reforma psiquiátrica em nossos cursos, nem a instrumentalizar o profissional com tecnologias cada vez mais sofisticadas, tampouco a abrir mais e mais postos de trabalho na rede assistencial. Huning e Guareschi dizem com precisão que:

Huning e Guareschi (2009) dizem com precisão que:

Reinventar práticas psi não diz respeito necessariamente a inventar outros métodos de intervenção, mas a introduzir outros modos de interrogar e outras interrogações, entre elas, o que pretendemos e quais as implicações de nossa prática, daquilo que afirmamos como verdades sobre os que tomamos como nossos sujeitos-objetos (p. 179)

Na verdade, apostamos em uma formação em que os psicólogos possam, minimamente, realizar leituras e análises conjunturais a respeito das necessidades sociais e de saúde da população, proceder à escuta e à intervenção sobre os processos psicológicos e psicossociais mobilizados pelas condições de vida e projetos de futuro da população, além da capacidade de articulação com as redes de serviços para operar práticas de cuidado mais integradas em saúde.

Talvez assim possamos construir, a partir das formações acadêmicas e profissionais dos psicólogos, modos de atuação e processos de trabalho mais democráticos e criativos, dotando os trabalhadores de maior capacidade de acolher e de resolver necessidades de saúde e de ampliar o seu grau de realização. Em outras palavras, nota-se uma outra relação do profissional com o mundo, uma potência para determinar o indeterminado, para lidar com a contingência, não a eliminando, mas agindo sobre ela, fundando assim uma política inteiramente nova não ancorada na fraqueza e na impotência.

\section{Magda Dimenstein}

Mestre em Psicologia Clínica pela Pontifícia Universidade Católica do Rio de Janeiro e Doutora em Saúde de Psiquiatria da Universidade Federal do Rio de Janeioro. Professora titular da Universidade Federal do Rio Grande do Norte, Rio Grande do Norte - RN - Brasil.

E-mail: magda@ufrnet.br

\section{João Paulo Macedo}

Professor adjunto da Universidade Federal do Piauí, vinculado ao Departamento de Psicologia do campus Parnaíba. Psicólogo pela Faculdade Santo Agostinho, Mestre e Doutor em Psicologia pela Universidade Federal do Rio Grande do Norte. Parnaíba - PI - Brasil.

E-mail: jpmacedo@ufpi.edu.br

Endereço para envio de correspondência:

Universidade Federal do Rio Grande do Norte - UFRN, Programa de Pós-Graduação em Psicologia Departamento de Psicologia, sala 610

Campus Universitário - Natal, Rio Grande do Norte - Brasil. CEP: 59078-970 
Referências
Ayres, J. R. C. M. (2001). Sujeito, intersubjetividade e práticas de saúde. Ciência e Saúde Coletiva, 6(1),63-72. Recuperado em 18 abril, 2012, de http://www.scielo.br/pdf/csc/v6n1/7025.pdf

Benevides, R. (2005). A psicologia e o Sistema Único de Saúde: quais interfaces? Psicologia \& Sociedade, 17(2), 21-25. Recuperado em 18 abril, 2012, de www.scielo.br/pdf/psoc/ v17n2/27040.pdf

Bernardes, A. G. (2010). Psicologia e o sistema indivíduo de saúde. In N. M. F. Guareschi, A. Scislesk, C. Reis, G. Dhein \& M. A. Azambuja (Orgs). Psicologia, formação, políticas e produção em saúde (pp. 206-220). Porto Alegre: EDIPUCRS

Böing, E. (2009). O psicólogo na atenção básica: uma incursão pelas políticas públicas de saúde brasileiras. Dissertação de mestrado. Programa de Pós-Graduação em Psicologia Universidade Federal de Santa Catarina, Florianópolis, SC.

Brasil. Ministério da Saúde. (2011). Guia prático de matriciamento em saúde mental. Ministério da Saúde: Centro de Estudo e Pesquisa em Saúde Coletiva, Brasília, DF.

Brasil. Ministério da Saúde. Secretaria de Atenção à Saúde. Departamento de Atenção Básica. (2012). Autoavaliação para a melhoria do acesso e da qualidade da Atenção Básica. Brasília, DF: AMAQ Ministério da Saúde. Secretaria de Atenção à Saúde. Departamento de Atenção Básica. Ministério da Saúde,

Campos, G. W. S. (1994). Considerações sobre a arte e a ciência da mudança: revolução das coisas e reforma das pessoas. $\mathrm{O}$ caso da saúde. In L. C. Cecílio (Org.), Inventando a mudança na saúde (pp. 29-88). São Paulo: Hucitec.

Campos, G. W. S., Gutiérrez, A. C., Guerrero, A. V. P., \& Cunha, G. T. (2010). Reflexões sobre a atenção básica e a Estratégia de Saúde da Família. In G. W. S. Campos \& A. V. P. Guerrero (Orgs.), Manual de Práticas de Atenção Básica. Saúde ampliada e compartilhada (pp. 132-153). São Paulo: Aderaldo e Rothschild.

Ceccim, R. B. (2010). Residências em saúde: as muitas faces de uma especialização em área profissional integrada ao SUS. In A. P. Fajardo, C. M. F. Rocha \& V. L. Pasini (Orgs.), Residências em saúde. Fazeres e saberes na formação em saúde (pp. 17-22). Porto Alegre: Hospital Nossa Senhora da Conceição. (Brasil. Ministério da Saúde. Grupo Hospitalar Conceição (GHC).)

Conselho Federal de Psicologia, CFP (2006). I Fórum Nacional de Psicologia e Saúde Pública: contribuições técnicas e políticas para avançar o SUS. Brasília, DF.

Ferreira, S. R., \& Olschowsky, A. (2010). Residências: uma modalidade de ensino. In A. P. Fajardo, C. M. F. Rocha \& V. L. Pasini (Orgs.), Residências em saúde. Fazeres e saberes na formação em saúde (pp. 23-34), Porto Alegre: Hospital Nossa Senhora da Conceição. (Brasil. Ministério da Saúde. Grupo Hospitalar Conceição (GHC).)

Feuerwerker, L. (2005). Modelos técnicos assistenciais, gestão e organização do trabalho em saúde: nada é indiferente no processo de luta para a consolidação do SUS. Interface. Comunicação, saúde e educação, 18(9), 489-506. Recuperado em 18 abril, 2012, de www.scielo.br/pdf/icse/v9n18/ a03v9n18.pdf

Figueiredo, M. D., \& Furlan, P. G. (2010). O subjetivo e o sociocultural na produção de saúde e autonomia. In G. W. S. Campos \& A. V. P. Guerrero (Orgs.), Manual de Práticas de Atenção Básica. Saúde ampliada e compartilhada. (pp. 154178). São Paulo: Aderaldo e Rothschild.

Fleury, S. (2009). Reforma sanitária brasileira: dilemas entre o instituinte e o instituído. Ciência \& Saúde Coletiva, 14(3), 743752. Recuperado em 18 abril, 2012, de http://www.scielo.br/ $\mathrm{pdf} / \mathrm{csc} / \mathrm{v} 14 \mathrm{n} 3 / 10 . \mathrm{pdf}$

Fuganti, L. (2008). Saúde, desejo e pensamento. São Paulo: Aderaldo e Rothschild. Ed. Linha de Fuga.

Gil, A. C. (1985). O psicólogo e sua ideologia. Psicologia: Ciência e Profissão, 5(1), 13-17. Recuperado em 18 abril, 2012, de www.pepsic.bvsalud.org/pdf/pcp/v5n1/05.pd

Guareschi, N. M. F., Scislesk, A., Reis, C., Dhein, G., \& Azambuja M. A. (Orgs.). (2010). Psicologia, formação, políticas e produção em saúde. Porto Alegre: EDIPUCRS.

Huning, S. M., \& Guareschi, N. M. F. (2009). Efeito Foucault: desacomodar a psicologia. In N. M. F. Guareschi \& S. M Huning (Orgs.), Foucault e a psicologia (pp. 159-182). Porto Alegre: EDIPUCRS

Instituto Brasileiro de Geografia e Estatística - IBGE. (2009). Pesquisa de assistência médico-sanitária (AMS). Brasília, DF: IBGE.

Macedo, J. P., \& Dimenstein, M. (2011). Expansão e interiorização da psicologia: reorganização dos saberes e poderes na atualidade. Psicologia: Ciência e Profissão, 31(2), 296-313. Recuperado em 18 abril, 2012, de www.scielo.br/pdf/pcp/ v31n2/v31n2a08.pdf

Machado, M. H., Médici, A. C., Nogueira, R. P., \& Girardi, S. N. (1992). O mercado de trabalho em saúde no Brasil: estrutura e conjuntura. Rio de Janeiro: ENSP.

Machado, M. H. (2006). Trabalhadores de saúde e sua trajetória na reforma sanitária. In Ministério da Saúde (Org.), Caderno RH Espaço Saúde, (3, pp.13-28). Brasília, DF: Auto

Medeiros Junior, A., Liberalino, F. N., \& Costa, N. D. L. (Orgs.) (2011). Caminhos da tutoria e aprendizagem em saúde e cidadania (3a ed.). Natal, RN: EDUFRN.

Mendes, E. V. (1994) As políticas de saúde no Brasil nos anos 80: a conformação da reforma sanitária e a construção da hegemonia do projeto neoliberal. In E. V. Mendes, $O$ distrito sanitário (pp.19-94). Rio de Janeiro: Relume-Dumará.

Neves, C. A., \& Rollo, A. (2006). Acolhimento nas práticas de produção de saúde. Núcleo Técnico da Política Nacional de Humanização (2a. ed.). Brasília-DF: Ministério da Saúde.

Oliveira, C., \& Guareschi, N. M. F. (2010). Residência multiprofissional em saúde: brechas para novas formas de conhecimento? In N. M. F. Guareschi, A. Scislesk, C. Reis, G. Dhein \& M. A. Azambuja. (Orgs.), Psicologia, formação, políticas e produção em saúde (pp. 99-117). Porto Alegre: EDIPUCRS

Paim, J. S. (2008). A reforma sanitária brasileira e o Sistema Único de Saúde: dialogando com hipóteses concorrentes. Physis. Revista de Saúde Coletiva, 18(4), 625-644. Recuperado em 18 abril, 2012, de http://www.scielo.br/pdf/physis/v18n4/ v18n4a03.pdf

Paim, J. S., \& Almeida Filho, N. (2000). A crise da saúde pública e a utopia da saúde coletiva. Salvador, BA: Casa da Saúde.

Scarcelli, I. R., \& Junqueira, V. (2011). O SUS como desafio para a formação em psicologia. Psicologia: Ciência e Profissão, 31(2), 340-357. Recuperado em 18 abril, 2012, de http://www.scielo. br/pdf/pcp/v31n2/v31n2a11.pdf

Silva, Q. T. A., \& Caballero, R. M. S. (2010). A micropolítica da formação professional na produção do cuidado: devirresidência. In A. P. Fajardo, C. M. F. Rocha \& V. L. Pasini (Orgs.), Residências em saúde. Fazeres e saberes na formação em saúde (pp. 61-74). Porto Alegre: Hospital Nossa Senhora da Conceição. (Brasil. Ministério da Saúde. Grupo Hospitalar Conceição (GHC).)

Yamamoto, O. H. (2007). Políticas sociais, "terceiro setor" e "compromisso social": perspectivas e limites do trabalho do psicólogo. Psicologia \& Sociedade, 18(1), 30-37. Recuperado em 18 abril, 2012, de http://www.scielo.br/pdf/psoc/v19n1/ a05v19n1.pdf 